\title{
Entre similitudes et métaphores. Amplification et optique dans la prédication en France (v. 1600-1670)
}

\section{Florent Libral}

\section{(2) OpenEdition Journals}

Édition électronique

URL : http://journals.openedition.org/rhetorique/351

DOI : 10.4000/rhetorique.351

ISSN : 2270-6909

Éditeur

UGA Éditions/Université Grenoble Alpes

Édition imprimée

ISBN : 978-2-84310-292-9

Référence électronique

Florent Libral, «Entre similitudes et métaphores. Amplification et optique dans la prédication en France (v. 1600-1670) », Exercices de rhétorique [En ligne], 4 | 2014, mis en ligne le 02 décembre 2014, consulté le 12 septembre 2020. URL : http://journals.openedition.org/rhetorique/351 ; DOI : https:// doi.org/10.4000/rhetorique.351

Ce document a été généré automatiquement le 12 septembre 2020.

\section{c) (1) (2)}

Les contenus de la revue Exercices de rhétorique sont mis à disposition selon les termes de la Licence Creative Commons Attribution - Pas d'Utilisation Commerciale - Partage dans les Mêmes Conditions 4.0 International. 


\title{
Entre similitudes et métaphores. Amplification et optique dans la prédication en France (v. 1600-1670)
}

\author{
Florent Libral
}

1 La présente étude, consacrée à la prédication post-tridentine française, se propose de repenser de concert trois notions : l'amplification, les formes comparatives ainsi que la science optique, prise en elle-même autant que dans ses applications à la perspective. Ce trio peut sembler à première vue insolite, mais les trois champs sont étroitement intriqués dans la Chaire, au sein de ce que l'on pourrait appeler une véritable rhétorique sacrée de l'optique. En premier lieu, les liens entre amplification et pensée comparative sont solidement attestés. À côté de l'amplificatio per congeriem et de l' amplificatio per ratiocinationem, l'amplificatio per comparationem est reconnue depuis l'Antiquité comme une des modalités essentielles de cette pratique textuelle ${ }^{1}$. Par ailleurs, dans les prédications d'après 1600 , la science de la vision inspire des images frappantes. Parmi elles se démarquent par leur fréquence celles qui comparent Dieu à la lumière, la connaissance qu'on en prend à la perception visuelle ; leur mise en œuvre entre dans le cadre canonique de l'amplification par comparaison qui va généralement du plus petit au plus grand; on passe ainsi de la créature au Créateur pour valoriser ce dernier, de l'œil du corps à celui de l'âme pour affirmer l'importance du second.

Or, deux formes comparatives fondées sur l'optique acquièrent une place maitresse dans l'éloquence post-tridentine, entre 1600 et 1670 environ. Dès 1600 , on note la vogue des «doctes similitudes», de longues comparaisons binaires qui développent dans un style abondant et érudit l'image du soleil et de la lumière divins ; Pierre de Besse, JeanPierre Camus ou encore Gaspard de Séguiran ${ }^{2}$ connaissent le succès vers 1600-1620 en pratiquant assidûment le lieu de la similitude. Vers 1620 , on voit apparaître un procédé apparemment contraire, celui de l'amplification par défaut, fondé sur des métaphores souvent contrastées, qui jouent sur l'opposition de la lumière et des ténèbres pour dire le Dieu incompréhensible; André de l'Auge, Étienne Molinier, Jean Le Jeune, Léon de Saint-Jean ${ }^{3}$ enrichissent leurs sermons de tels traits conceptistes. Ces éléments 
montrent que l'amplification par comparaison, qu'elle s'exerce par excès dans la similitude, ou par défaut au moyen de la métaphore pointue, joue un rôle essentiel dans la chaire française d'avant 1650, comme Peter Bayley l'avait justement noté4. Toutefois, les modalités de cette amplification semblent s'opposer du tout au tout, entre un héritage cicéronien des doctes similitudes en perte de vitesse dès les années 1630, et le courant des métaphores pointues qui émerge vers 1620 , plus sénéquien et lipsien ${ }^{5}$. Et bien que l'esthétique de la copia, comme celle de la pointe, ait été répudiée par le classicisme français au profit d'un atticisme tempéré, c'est bien une similitude que l'on retrouve dans l'anamorphose du Carême du Louvre de Jacques-Bénigne Bossuet (1662) :

Quand je considère en moi-même la disposition des choses humaines, confuse, inégale, irrégulière, je la compare souvent à certains tableaux, que l'on montre assez ordinairement dans les bibliothèques des curieux comme un jeu de la perspective. La première vue ne nous montre que des traits informes et un mélange confus de couleurs, qui semble être ou l'essai de quelque apprenti, ou le jeu de quelque enfant, plutôt que l'ouvrage d'une main savante. Mais aussitôt que celui qui sait le secret vous fait regarder par un certain endroit, aussitôt, toutes les lignes inégales venant à se ramasser d'une certaine façon dans votre vue, toute la confusion se démêle, et vous voyez paraitre un visage avec ses linéaments et ses proportions, où il n'y avait auparavant aucune apparence de forme humaine. C'est, ce me semble, Messieurs, une image assez naturelle du monde, de sa confusion apparente et de sa justesse cachée, que nous ne pouvons jamais remarquer qu'en le regardant par un certain point que la foi en Jésus-Christ nous découvre ${ }^{6}$.

Tout porte à croire que le premier xvII ${ }^{\mathrm{e}}$ siècle s'est englué dans des excès quantitatifs, en opposition avec le style de la varietas et avec l'atticisme modéré appelés à triompher ${ }^{7}$, mais les prédicateurs d'alors ont pourtant transmis à leurs successeurs immédiats une logique comparative fonctionnelle, fondée sur le dialogue avec l'optique. Il faudrait donc comprendre comment la réflexion et les expérimentations du premier XVII siècle concernant l'amplificatio per comparationem peuvent être répudiées à l'aube de l'ère classique, et en même temps continuer à inspirer aux auteurs du siècle de Louis le Grand leurs belles réussites oratoires sur les mêmes thèmes.

Si les doctes similitudes comme les métaphores sont des modes d'amplification opposés, mais également critiqués du fait de leur démesure (le trop ou le trop peu), il n'en reste pas moins que ce n'est pas tant par leur forme, mais par leur logique commune que ces formes comparatives ont été influentes: elles instillent dans l'amplification un principe essentiel de dissemblance entre le visible et l'invisible. Ceci permet de percevoir au centre de toute la période - jusqu'en 1670 environ - une idéephare : l'amplification est un instrument optique destiné à provoquer une conversion intégrale du regard de l'âme, le faisant passer de son aveuglement originel à la contemplation du Dieu caché.

\section{Deux modes d'amplification opposés, similitude et métaphore}

4 A priori, le début du siècle présente deux modes d'amplification fort différents, mais tout aussi extrêmes sur le plan quantitatif, ce qui les conduit à disparaître progressivement, en raison même de leur radicalité formelle. 


\section{Amplification par l'excès. Les similitudes lumineuses}

5 La similitude lumineuse se définit comme un rapprochement de Dieu avec le soleil et avec la lumière, sur la foi de caractéristiques ou d'affections communes. Le célèbre prédicateur jésuite Gaspard de Séguiran explique en 1612 que les propriétés du soleil "se retrouvent en Dieu en un degré plus relevé ${ }^{8}$ ". La similitude lumineuse ainsi théorisée est conforme à la théorie de l'amplificatio per comparationem, puisqu'elle va du plus petit au plus grand, du familier à l'inconnu (Quintilien, Richesource ${ }^{9}$ ) ; ce type de fonctionnement permet de faire valoir la supériorité du comparé sur le comparant, comme de le rendre moins obscur. Voici l'une de ces similitudes, tirée d'un sermon de Pierre de Besse :

Le Soleil en roulant tous les jours d'Orient en Occident, par ce mouvement admirable, sans s'alterer aucunement, sans changer de place nullement : il produict icy bas une infinité de choses: et ceste production ne luy apporte aucune nouveauté, mais si faict bien aux choses qui sont produictes. Il en faut croire de mesme de ce grand Dieu eternel, lequel roulant de toute eternité dans les flancs immenses de son essence : en ce mouvement surnaturel de la creation, a produit et mis aux champs une infinité de creatures, sans que leur production luy ayt porté aucune mutation, changement, ou autre façon d'estre : tout cela regarde seulement les creatures ${ }^{10}$.

L'immutabilité $\mathrm{du}$ soleil et sa fécondité se trouvent rapprochées de leurs correspondants divins. Ainsi que le montre cet extrait, et que le souligne Nicolas Caussin ${ }^{11}$, la similitude n'est pas un simple artifice de rhétorique, mais une révélation des médiations sensibles du divin inscrites dans la nature, parmi lesquelles la lumière occupe une place éminente.

6 Si elle est un moyen d'amplifier le discours, la similitude peut elle-même faire l'objet d'une amplificatio et ainsi se démultiplier. Dans son De copia, Érasme indique la voie royale pour développer la parabolè-similitudo, à savoir multiplier les traits de correspondance entre le comparant et le comparé ; François de Sales écrit pour sa part que toute métaphore biblique peut être amplifiée, selon ce procédé, en vaste similitude $^{12}$. Dans la pratique, cette théorie permet aux prédicateurs du début du XVII siècle de fonder l'invention de tout un discours sur la logique de la similitude entre Dieu et la lumière ; ainsi, une courte citation extraite du récit de la Transfiguration ( $E t$ resplenduit facies ejus sicut Sol, vestimenta autem ejus facta sunt alba sicut nix) permet à Séguiran d'amplifier dans un sermon du Carême l'analogie entre le Christ et la lumière, pour expliquer la génération éternelle du Verbe et l'éclat visible du Christ transfiguré aux yeux des Apôtres $^{13}$. Pierre de Besse, autre ténor de la chaire d'alors, opère un parallèle détaillé entre le Christ et le soleil à partir de l'Évangile de Jean (Ego sum lux mundi, qui sequitur me, non ambulat in tenebris); son sermon consiste à accumuler des justifications du rapprochement ("produisons d'autres conceptions ", "approchons plus près »), dans une structure qui fait succéder aux propriétés du soleil divin celles de ses lumières spirituelles, la grâce par exemple ${ }^{14}$. Ces discours semblent bâtis sur une pratique répertoriée dès l'Antiquité, celle de la similitudo per conlationem (« en parallèle ») des traités ${ }^{15}$; la fonction de ce type de similitude est de faire voir les choses (ante oculos poner ${ }^{16}$ ). Nulle étrangeté à ce que des éléments de visualisation viennent se couler assez exactement dans le moule de la similitude visuelle, comme dans cet autre sermon où Besse traite du Jugement dernier :

Il est certain que l'air est tout remply de certaines petites choses que les

Philosophes ont accoustumé d'appeler athomes, desquelles toutes les sales, 
chambres et cabinets d'une maison sont plaines, et neantmoins elles sont invisibles, et nostre veuë ne les peut descouvrir, ainsi tout aussi tost que les rayons penetrans de ce flambeau de la nature donnent à travers une vitre, ou les fentes d'une porte on les voit voltiger en grande quantité de toutes parts. Nos peschez ne sont rien que des athomes, de pures privations, qui volent de la concupiscence à la raison, et de la raison à la concupiscence, Caro concupiscit adversus spiritum, et spiritus adversus carnem [" la chair a des désirs contraires à ceux de l'esprit, et l'esprit en a de contraires à ceux de la chair»], et les chambres, sales, cabinets, sentiments, facultez, et recoins de nos ames en sont tous plains, et cependant en ceste vie on ne les peut voir, mais en ce clair jour du Jugement tout aussi tost que le brillant soleil de justice aura donné à travers les vitres de nos corps, et les fautes de nos consciences, on verra incontinent un'infinité de vices ${ }^{17}$.

La dimension descriptive de cet extrait apparaît tant dans l'ancrage spatial - la maison aux pièces multiples évoque quasiment le château de l'âme thérésien - que dans la dimension narrative - les atomes des péchés sont invisibles, puis apparaissent. L'hypotypose se trahit encore par les notations visuelles, à travers l'évocation du «flambeau », de la « vitre ». Chez des auteurs plus asianistes encore que le prédicateur de Condé, cette tendance est accentuée par l'influence de la rhétorique des peintures issue de Philostrate ${ }^{18}$.

7 Toutefois, la similitude ainsi dilatée dans la logique d'une abondance purement verbale peut apparaître mécanique, voire sophistique et vide. Un autre moyen de l'amplifier, en lui conférant cette fois-ci un certain poids intellectuel, consiste à y injecter une forme de savoir encyclopédique. Pour le prouver, il suffit de considérer le parallèle des péchés et des atomes qu'opère Pierre de Besse sous un nouveau jour. Derrière l'apparence d'un simple tableau se cachent de solides garants théologiques comme philosophiques, évoqués par exemple dans cette citation qui le suit immédiatement :

S. Augustin fonde ceste similitude, fiet enim (inquit) virtute divina ut cuique opera sua vel bona, vel mala mentis intuitu mira celeritate cernantur [" une vertu divine, par laquelle chacun se ressouviendra de toutes ses œuvres, tant bonnes que mauvaises, et elles lui seront toutes présentées en un instant $]^{19}$.

Une autorité patristique du premier ordre est donc mobilisée pour relier la thématique du regard au Jugement dernier, tandis que la description des atomes elle-même renvoie au De rerum natura de Lucrèce ${ }^{20}$. Le prédicateur se fonde sur l'héritage d'un humanisme pieux assimilant l'héritage antique, qu'il soit chrétien ou non. Loin d'être une simple hypotypose relevant de la copia verborum, la similitude de Pierre de Besse se rattache donc de plein droit à la copia rerum. Plus généralement, dans le cas de la similitude lumineuse, c'est tout le savoir scolastique sur la lumière, ainsi que des conceptions occultes issues du paracelsisme et de la kabbale qui sont convoqués pour renforcer l'image biblique du Dieu lumière ${ }^{21}$.

\section{Amplification par défaut. Style abrégé, similitude et métaphore}

8 Pourtant, l'amplification ne se limite pas à l'abondance, mais suit aussi les voies de la brièveté emphatique, sous l'étendard sénéquien; les modalités en sont doubles, la similitude abrégée alternant avec la métaphore. Dans le premier cas, la similitude visuelle est réduite à sa plus simple expression, selon la logique de la similitudo per brevitatem décrite dans les traités ${ }^{22}$, et pratiquée ici par Gaspard de Séguiran :

Dieu est peu cogneu, et le Soleil peu veu : Dieu est le souverain intelligible : et le

Soleil le souverain visible, Dieu est appelé par l'Apostre sainct Jacques Pater 
luminum, le Soleil aussi est le pere de toutes les lumieres du monde. Le Pere eternel est fœcond en sa nature, ainsi le Soleil est fœcond en lumiere ${ }^{23}$.

Les parallèles sont exprimés le plus simplement possible ${ }^{24}$, l'asyndète se substituant aux pesants « de même que ». Cette structure allégée permet d'éviter les défauts d'une trop grande copia verborum et de concentrer au maximum l'expression, en même temps que de démultiplier un motif unique sous différentes facettes; gage de plus de clarté (apertius dicendi causa), la concentration de l'expression est de nature à capter l'attention de l'auditeur.

9 Un échelon supérieur est gravi dans l'atticisme avec la métaphore. Similitude contractée en un mot d'après les traités, elle est souvent sous-tendue par une analogie complète entre quatre termes (A est à $\mathrm{B}$ ce que $\mathrm{C}$ est à $\mathrm{D}$ ), ainsi que le recommande Aristote dans la Rhétorique $e^{25}$, ce qui la dote d'une réelle profondeur et en fait tout autre chose qu'un simple ornement. Ainsi, dans une amplification des trois mots qui constituent toute la prière de Bartimée, l'aveugle évangélique (Marc, x, 52), Étienne Molinier, célèbre prédicateur toulousain, use de plusieurs métaphores en réseau :

C'est pourquoy il seroit à souhaiter que cette priere de l'aveugle de nostre Evangile, Dominus ut videam, fut frequente tous les jours en nos cœurs, et en nos bouches, non au sens que cest aveugle l'entendoit pour demander à Dieu la lumiere de la veuë corporelle, mais en un sens plus haut, et digne de ceux qui aspirent à Dieu, pour le supplier de donner à l'œil de nostre intellect la lumiere spirituelle de sa grace, et ne permettre pas que cest œil si necessaire pour le voir, et le contempler se déprave par les erreurs et tombe dans l'aveuglement, porte de la reprobation, Domine ut videam, Seigneur donnez-moy cette veüe interieure, dont vous mesmes estes l'object qu'elle regarde, et le Soleil qui l'illumine ; Et vous saincte Vierge Aurore, et Mere du Soleil qui a porté au monde la lumiere pour éclairer nostre œil interieur, impetrés-la par vos suffrages à celuy qui en veut discourir pour la cognoistre luymesme, et la communique à ses Auditeurs ${ }^{26}$.

10 À la métaphore de la lumière de la grâce répond celle de l'œil spirituel censé la recevoir, mais qui peut devenir aveugle du fait du péché, et donc perdre de vue la clarté divine. Derrière son apparence anodine, le réseau métaphorique divulgue, tout en la dissimulant, une théorie complète de la grâce. En un mot, la métaphore concilie la légèreté formelle (brevitas) et la portée suggestive de l'insinuation ${ }^{27}$ (emphasis), d'autant plus marquante en ce passage que Molinier développe par ailleurs de longues similitudes. Cette force attique de la métaphore est portée à son point culminant dans la métaphore pointue, qui fait une entrée dans le sermon aux alentours des années 1620, par exemple pour expliquer le mystère de l'Incarnation, qui concilie la clarté du divin et l'obscurité de l'humanité. La pointe est fondée sur un paradoxe, une contradiction, par exemple lorsqu'André de l'Auge évoque une « lumière claire brune » pour signifier la foi des croyants en la béatitude à venir ${ }^{28}$. Le clair-obscur évoque alors l'espérance de la gloire éclatante après cette vie ténébreuse; il résulte d'une telle stratégie rhétorique une autre obscurité, cette fois-ci au sens stylistique, qui est une des voies privilégiées de l'amplification, quoique directement opposée à la tendance qui voudrait limiter cette dernière à un accroissement du matériel lexical ${ }^{29}$.

11 Si dans sa forme la métaphore peut être conçue comme une similitude abrégée, sa logique est pourtant bien différente. La similitude ambitionne, par l'effet conjoint de la copia verborum asianiste et de l'érudition encyclopédique cicéronienne, de recréer l'univers à l'aide des mots pour le raccorder à son principe divin unique et caché, le soleil divin; à l'inverse, la métaphore propose un itinéraire spirituel bien plus 
troublant. Jean Le Jeune, infatigable prédicateur itinérant, en fournit un exemple magistral lorsqu'il évoque la naissance du Verbe dans l'Éternité :

Il est vrai que cette naissance est une lumiere, elle est in Splendoribus Sanctorum. Mais cette lumiere est si brillante, si éclatante qu'elle nous éblouït les yeux; nous n'y voyons rien non plus qu'en plein minuit, non plus que le hibou qui par la foiblesse de sa veuë est en la nuit au milieu du jour. Ne parlons donc plus de ce que nous ne pouvons comprendre, ne soyons pas si temeraires que de vouloir approcher cette lumiere inaccessible, de vouloir expliquer ce que les Anges mêmes dans la clarté de la gloire ne peuvent pas comprendre ${ }^{30}$.

12 Ce trait montre que la métaphore se conjugue à d'autres traits du style coupé. Elle s'allie aux sentences bibliques intégrées au texte, et choisies du fait de leur opposition. La splendeur qu'est le Verbe aux yeux des saints selon le Psaume 109 de la Vulgate (verset 3) contraste avec la «lumière inaccessible ${ }^{31}$ » de l'épître paulinienne, qui le cache aux yeux des mortels. Le procédé vise à désorienter l'auditeur, à lui proposer un parcours biblique empreint d'une cohérence qui n'est pas celle de la raison raisonnante, mais celle de la sagesse divine. Le point de vue des saints souligne par contraste l'incompréhension humaine; or, ces changements de focale sont, pour Christian Mouchel, un des caractères principaux de l'amplification sénéquienne dans la prédication, qui permettent de convaincre en faisant miroiter les différents aspects d'une même idée ${ }^{32}$.

13 Concluons ce premier parcours en insistant sur l'insuffisance et l'interdépendance de chacune de ces deux pratiques: la similitude ne peut se passer de l'agrément de la métaphore, quand celle-ci ne suffit pas toujours pour enseigner. En elles-mêmes, la similitude lumineuse comme la métaphore font preuve de répétitivité sur un plan purement littéraire; dans leur ancrage érudit, elles véhiculent un savoir optique faiblement rénové. Enfin, comparaison n'est pas raison; depuis Rodolphe Agricola, les traités soulignent que similitudes et métaphores servent plus à enseigner qu'à prouver ${ }^{33}$. Rien d'étonnant à ce que ces défauts aient fini par lasser. La similitude se heurte au mépris des galants pour la pédanterie et à leur volonté de variété ${ }^{34}$; la métaphore tombe sous le coup des attaques de Bouhours et de Boileau contre le style serré assimilé à l'obscurité et à l'équivoque ${ }^{35}$. Métaphore et similitude survivent bien après 1660 , mais sous une forme galante, en ayant souvent perdu leur dimension savante. Ainsi, il semble possible de reconduire partiellement un schéma évolutionniste qui verrait deux formes excessives et baroques amendées - ou affadies, selon le point de vue que l'on adopte - par la juste mesure classique.

\section{Du principe de dissimilitude dans l'amplification des formes comparatives}

Malgré tout ce qui les oppose, similitudes et métaphores évoluent en un sens absolument convergent, qui insiste de plus en plus, au fil des années, sur la dissemblance entre tout comparant et le comparé divin. En effet, dans la théorie rhétorique depuis Quintilien ${ }^{36}$, et encore pour Léon de Saint-Jean (1655), toute similitude s'accompagne d'un principe de dissimilitude :

Cela présupposé, il est tres facile de faire revenir toutes les matieres à tout discours: et parler de toutes choses à propos de toutes choses, sans jamais extravaguer hors de propos. Et cela en deux differantes manieres. Par convenances, rapports, comparaisons et similitudes: tout au contraire par dissamblances, 
disconvenances, anthitezes et dissimilitudes. Si bien que les ayant treuvées, il les

faut explicquer, et preuver : puis les applicquer, et amplifier leur application ${ }^{37}$.

Pour Léon de Saint-Jean, il est explicitement question d'amplifier ces similitudes et dissimilitudes afin de bâtir un sermon. Une fois posé ce principe, il reste à comprendre comment il est mis en œuvre à la fois dans l'amplification copieuse et dans celle qui est placée sous le signe de l'emphasis. L'élément de dissonance est évident dans les métaphores dès le départ; en revanche, il s'introduit dans les similitudes de manière progressive.

\section{Un principe présent dans les métaphores dès 1620}

15 Le principe de dissemblance est inscrit de manière essentielle dans les métaphores pointues qui jouent sur le paradoxe, la fusion des opposés signifiant la relativité du savoir mondain face à la sagesse divine. Le procédé le plus frappant qu'utilise le style bref pour amplifier les similitudes est le choc des antonymes; le rhéteur Domenico Colonia parle à ce sujet d'une amplification per conflictionem contrariorum ${ }^{38}$. Les divers synonymes des ténèbres et de la lumière sont complémentaires pour suggérer la double nature du Dieu visible et invisible. Léon de Saint-Jean, célèbre prédicateur carme à la Cour du jeune Louis XIV, affectionne particulièrement le procédé :

Pour penetrer le fond de ces grandes veritez, comprenez avec attantion; que JESUs est tout à la fois et lumieres, et tenebres. Lucem habitat inacessibilem, voila les lumieres. Et voicy les tenebres, posuit tenebras latibulum suum. Il est engendré dans les splandeurs des Saints, in splendoribus sanctorum. Cela n'est-il pas plein de gloire, et de magnificence? Mais il est engendré devant l'étoile du matin, ante Luciferum, donc devant le jour, dans la nuit, dans les tenebres du Sein, à qui l'Ecriture donne un nom d'obscurité ; ex utero, ex vulva ante Luciferum genui te $e^{39}$.

La métaphore in prcesentia souligne ici l'association paradoxale des ténèbres et de la lumière dans la personne du Christ, en se fondant sur la lettre même de la Bible, qui évoque la "lumière inaccessible » dont il est si difficile de définir la nature. Il s'ensuit ainsi que l'ordre de la nature, dans lequel les antonymes sont opposés, s'efface devant la réflexion théologique, dans lequel leur combinaison fait sens :

Je remarque d'abord, mes bien-aimez Auditeurs, qu'il y a cete notable differance entre les deux mysteres de la Creation, et de l'Incarnation, qu'en celle-là Dieu le Createur a separé la lumiere d'avec les Tenebres, que dans le second il a uny ces deux qualitez contraires et opposées. Dans le premier il a méme donné des noms differans à l'un et à l'autre, appelans la lumiere le jour, et les tenebres la nuit. Dans le second l'on peut dire qu'il a fait liaison de tous les deux sous un méme nom, nyktèmeron [...]. Il en fait une imitation de la Divinité, qui tout à la fois habite une lumiere inaccessible, et s'enferme dans l'epaisseur des tenebres ${ }^{40}$.

Qu'il s'agisse ici de l'antithèse ${ }^{41}$ entre "ténèbres" et "lumières ", ou ailleurs de l'oxymore (« l'eclipse de la Passion eclaire l'Esprit de ce Philosophe $\left.{ }^{42} »\right)$, les métaphores mettent en œuvre le paradoxe dans une logique sénéquienne; elles contribuent donc à dynamiter les catégories reçues de la perception et de la logique, pour évoquer un Dieu qui dépasse le principe de contradiction.

Pourtant, ces obscurités stylistiques, qui s'intègrent très bien dans la poésie mystique comme celle de Claude Hopil, où brillent les rayons ténébreux et autres soleils cachés ${ }^{43}$, semblent poser problème dans l'éloquence de la chaire où il s'agit de convaincre rationnellement. Nicolas Caussin, par exemple, réserve la métaphore trop obscure aux poètes, et recommande la similitude aux prédicateurs ${ }^{44}$. Dans le cas de Léon de SaintJean, cette dichotomie a pourtant un sens dialectique, qui est d'affirmer la relativité 
essentielle de toute connaissance face à l'Être infini. Ainsi, évoquant les mystères divins, Léon indique que " ces Tenebres ne sont point des privations de Lumiere, mais des effusions et des abondances trop grandes ${ }^{45}$ ». En un mot, ce qui est ténèbres pour la raison est lumière pour la foi et inversement; la contradiction s'explique par le décalage entre la sagesse humaine et la «folie de la croix » paulinienne, plus apte à percer les mystères divins. Léon rattache cette valeur propédeutique de la métaphore à des philosophes et logiciens comme Raymond Lulle ou Nicolas de $\mathrm{Cues}^{46}$; toutefois, sur un plan stylistique, c'est bien un sénéquisme formel qui est en jeu, lorsque le bénédictin Jacques Biroat ${ }^{47}$ use d'un semblable clair-obscur pour suggérer la double nature de la foi :

Et c'est icy qu'il faut resoudre une importante question de la Theologie, et accorder les sentimens differents des Docteurs touchant la nature de la foy. Quelques-uns disent qu'elle est obscure ; quelques autres asseurent qu'elle a beaucoup de clarté et d'evidence. Ceux-là veulent qu'elle soit apparemment contraire à la raison ; ceux-cy qu'elle soit tres-conforme à ses lumieres. Joignons ensemble ces differentes opinions pour les accorder, disons que la foy est obscure et evidente, suivant les divers visages qu'elle a, et qu'elle ressemble à cette Colomne de nuée et de feu qui conduisoit les Israëlites dans le Desert, et que d'un costé elle a des nuées et des ombres, et de l'autre des feux et des lumieres ${ }^{48}$.

La foi se caractérise par les "divers visages qu'elle a » : autrement dit, la notion de point de vue est introduite dans ces lignes de manière particulièrement pertinente pour définir un mot. Il n'est pas d'obscurité en Dieu, si ce n'est aux yeux d'une l'humanité aveuglée par le péché ; la métaphore restaure donc la visibilité divine.

17 Ainsi, par-delà les références savantes et bibliques qu'elle mobilise, l'amplification par métaphore souligne dans sa logique essentielle qu'aucune image n'est adéquate à Dieu. Les ténèbres ne conviennent pas mieux que la lumière pour signifier l'être infini. Au Dieu cicéronien des similitudes, qui s'adresse à l'homme par le soleil, se substitue un Dieu sénéquien qui parle par emphasis, usant, selon Léon de Saint-Jean, d'un style énigmatique pour faire sortir l'homme des apories de la sagesse de la chair. Le prédicateur évoque la manière dont Denys l'Aréopagite se convertit à la suite de l'éclipse miraculeuse advenue lors de la Passion du Christ :

Ainsi une Enigme produit une Enigme, et l'eclipse de la Passion eclaire l'Esprit de ce Philosophe des lumieres de la foy. De voir, dit S. Chrysostome, des nobles, des riches, des savans se gagner par les persuasions de la noblesse, des richesses, de la sagesse; certes il n'y a ny enigme, ny difficulté, ni nouveauté. Mais tout le christianisme, pour parler avec mon Divin Maître, est une nouveauté, un paradoxe. Il ne parait que des tenebres, de l'obscurité, une eclipse. Le mot caché, c'est celuycy; un Dieu pert la vie sur un gibet, pour la donner à ses Ennemis et à ses Bourreaus : les tenebres de cete eclipse eclairent l'esprit d'un Platonicien, et d'un savant Philosophe et en font un humble Chretien. Occidet sol in meridie, et tenebrosas faciam terras in die hominis [" Le soleil se couchera en plein midi, et je couvrirai la terre de ténèbres lorsqu'elle devrait être plein de lumière $»]^{49}$.

On a ici l'exemple français d'une justification théologique de l'éloquence de la pointe, assez semblable à certaines idées d'Emmanuele Tesauro. Pour les deux auteurs, avant d'être une trouvaille de style, la métaphore pointue est le langage énigmatique de la divinité elle-même ${ }^{50}$ : per verbum in œnigmate. C'est que Léon, comme le rhéteur turinois, se situe dans une lignée lipsienne, selon laquelle toute image de Dieu est dissimilaire par nature ; il faut passer par le voile pour atteindre le Saint des Saints ${ }^{51}$. 


\section{L'intégration graduelle de la dissemblance dans les similitudes après 1630}

18 Une telle dialectique du paradoxe aurait pu être l'apanage des métaphores, car les similitudes ne traitent par définition que du semblable. Pourtant, certaines similitudes visuelles, notamment parce qu'elles s'appuient sur l'optique et la perspective contemporaines, insistent davantage sur la distance existant entre Dieu et le monde. Après les années de vogue des similitudes lumineuses, la logique de l'amplification d'une sympathie entre Dieu et le soleil recule, car aucune image, aussi brillante soitelle, ne peut représenter Dieu parfaitement, du fait de son infinité et de sa transcendance. Le capucin Thomas Giroult le disait déjà en 1611 dans un sermon consacré aux différentes similitudes du Verbe :

Ainsi voylà comme nous begayons, pour faire voir quelque chose du Verbe divin $d u$ Pere celeste, par les rapports et les analogies que nous en faisons avec le nostre: non qu'en toutes ces proportions, en toutes ces ressemblances, il n'y ait de la disproportion et de la dissemblance. Car comme disoit S. Basile, ce premier maistre de la vie religieuse, contre Eunomius, il est impossible de toute impossibilité, que l'on puisse entierement acquarrir [équarrir, adapter] les choses creées au createur, et que l'on puisse du tout assortir les choses viles et petites de cette terre aux choses divines et eternelles de ce grand Tout, non est, inquit, possibile, parva et vilia, divinis atque ceternis, ad unguem accomodari ["il n'est pas possible d'adapter avec exactitude de petites et insignifiantes réalités aux réalités divines et éternelles »] ${ }^{52}$.

Les sources théologiques d'une telle pensée de la dissimilitude sont multiples. Outre Saint Basile évoqué dans cet extrait, le Pseudo-Denys affirmait également l'imperfection de toute analogie, y compris de celle de la lumière ${ }^{53}$. Ce principe, oublié dans les années de gloire de la similitude lumineuse qui surexposait le soleil divin, revient en force à partir des années 1630 .

Pour exprimer cette transcendance absolue de Dieu, rétive à toute image, les similitudes amplifient l'idée de dissemblance ; elles soulignent le fossé qui existe entre le monde spirituel et le monde matériel. Ainsi en est-il de l'anamorphose catoptrique vue par le jésuite Joseph Filère, qui fournit, moins de trente ans avant le Carême $d u$ Louvre, une première occurrence importante du motif. Ce jeu de perspective consiste à montrer

une image, qui de premier abord paroît difforme et disproportionnée, quand on la regarde directement; elle se montre neanmoins tres-belle et bien proportionnée, quand on la voit par la reflexion du miroir cylindrique ${ }^{54}$.

Cette révélation d'une image par le miroir permet d'introduire, dans le comparé de cette similitude, une opposition fondamentale, cette fois-ci théologique, entre la nature prise en elle-même ou comme reflet du divin :

[Le pécheur] est semblable à celuy, qui sans savoir le dessein du peintre, de representer une image parfaite dans le miroir cylindrique, s'arrêteroit à regarder seulement les couleurs, qu'il a couchées sur le plan, qui ne donnent que peu de contentement à l'œil : parce que la principale piece de la beauté leur manque ; sçavoir est, la proportion, laquelle ne paroît pas, quand on les regarde directement. Mais l'homme sage les regarde d'un autre biais, et d'un aspet tout different. C'est dans Dieu, comme dans un miroir rond et cylindrique, pour recevoir la reflexion de toutes les lignes du plan de l'Univers, qui aboutissent à luy comme en leur centre; et c'est aussi dans luy, qu'il en voit resulter une image d'une parfaite beauté, toutes les perfections des choses creées n'étans que les couleurs et les lignes, pour representer en cette reflexion les excellences de la Divinitét 55 . 
Ainsi la similitude permet-elle, tout autant que la métaphore, de souligner l'opposition entre un point de vue erroné de la chair, et celui plus complet que restaure la sagesse en ramenant tout à Dieu. Si Filère n'est pas à proprement parler un sermonnaire, son expérimentation sur la similitude trouve un écho sous la plume des prédicateurs, qui sauront à sa suite moraliser en un sens proche les perspectives curieuses, comme l'oratorien Charles Hersent en $1644^{56}$.

Il faut noter que cette mutation se fait sous le signe d'une technique empruntée à l'optique et à la perspective, l'anamorphose. Le rapport à la science, loin d'être secondaire, influe donc sur l'évolution des similitudes, en leur permettant de valoriser le principe de dissemblance. Et ceci est possible précisément parce que la nouvelle science explique le fonctionnement d'instruments optiques ou catoptriques en termes d'inversion du visible, l'exemple canonique en étant la chambre noire. Voici comment la décrit le minime Pierre Blanchot (1635):

Or ce changement se fait par une croix naturelle, semblable à celle de S. André ; Car les especes des choses allant directement par ce pertuis, et passant outre jusques à ce qu'elles soient terminées sur quelque suject, elles se croisent et ont deux angles; l'un hors de la chambre, l'autre dedans. Tellement que l'espece et l'image de la teste qui estoit en haut hors de la chambre, se porte en bas au dedans, et l'espece des pieds semblablement qui dehors estoient en bas, au dedans paroist en haut; ce sont deux lignes qui se croisent et qui s'entrecoupent egallement. Or ce que la nature opere necessairement par l'emission de ces especes croisées, la grace le produict volontairement par la croix de Jesus qui change le ciel en terre, et la terre en ciel ; r'abaisse les choses eslevées, rehausse celles qui estoient abaissées ${ }^{57}$.

Ici, l'opposition de l'objet et de son image inversée permet de suggérer le contraste entre la tristesse des martyrs en cette vie et leur gloire dans l'au-delà. En somme, ce n'est rien moins que la « folie de la croix ${ }^{58}$ » paulinienne qui se trouve ainsi amplifiée par une image frappante.

\section{Évolution convergente des similitudes et des métaphores}

21 Ainsi, par-delà leurs divergences formelles, similitudes et métaphores semblent donc évoluer de manière convergente, alors que s'affirme l'insuffisance de toute image de l'Invisible. À la réduction du multiple à l'unité d'inspiration cicéronienne ${ }^{59}$, certains orateurs préfèrent désormais la pluralité des points de vue appelés à se réduire - ou non - à un seul. En effet, à l'origine, la similitude visait à surexposer l'objet divin en amplifiant sa comparaison avec la lumière ; cette logique est devenue caduque, pour des raisons complexes qui tiennent à la théorie classique du signe et au progrès des connaissances scientifiques, tout autant qu'à la volonté de rompre avec la réification d'une image qui pourrait devenir une idole ${ }^{60}$. Par conséquent, alors qu'en 1604 Pierre de Besse s'étonnait devant la surprenante "sympathie ${ }^{61}$ " - au sens d'une relation d'affinité cachée - qui existait entre Dieu et le soleil, en 1658, le jésuite Jean Grisel constate au contraire l'inadéquation profonde de l'analogie.

Il n'est pas, Messieurs, que vous ne sçachiez bien que Jesus Christ est quelquefois comparé au Soleil, mais il faut que vous sçachiez que cette comparaison a plus d'esclat que de verité. Je veux que cét astre ait du rapport en son lever avec la venuë de Jesus-Christ au monde, et que tout le temps qu'il est sur l'orison, la vitesse dont il fournit sa course, ait servy jadis à David pour exprimer la joye avec laquelle le Fils de Dieu a fourny la sienne : mais on ne peut dire qu'il luy ressemble en son couchant, puis que le Soleil semble lors se délasser du travail de sa course, en se couchant dans la mer : au lieu que Jesus Christ travaille plus que jamais, lors que cét 
Astre finit le jour, et qu'il applique ses divines mains à la cure de tous les malades qui luy sont presentez ${ }^{62}[. .$.$] .$

Le soleil divin laisse place au Dieu caché63, évoqué par des prédicateurs de toute tendance: dès 1627, le dominicain Charles Roussel montre la divinité cachée dans l'humanité du Christ comme dans un tableau énigmatique ${ }^{64}$ tandis que Léon de SaintJean l'invoque en 1661 devant les religieuses de Montmartre ${ }^{65}$. Le divin étant réputé difficile d'accès, l'amplification, faute de se réduire au silence, doit donc changer d'objet et se focaliser sur le sujet humain. Métaphore des ténèbres et métaphore des lumières expriment l'expérience subjective du croyant : ainsi, la conversion vécue par Denys l'Aréopagite symbolise pour Léon de Saint-Jean celle de l'humanité entière, et acquiert ainsi une valeur paradigmatique fondamentale. Le saint athénien de la légende a cru, en voyant l'impossible se produire - une éclipse de soleil au temps de la pleine lune. De même, l'homme doit avoir foi en l'incompréhensible.

\section{Les enjeux de l'amplification optique : restaurer la vérité}

Ainsi, l'évolution des rapports entre amplification par comparaison et optique semble se résumer à une recherche de l'équilibre entre ressemblance et dissemblance, ou pour le dire de manière imagée, " entre ombre et lumière ${ }^{66}$ ». Trop de clarté ferait perdre de vue la transcendance divine; trop d'obscurité désorienterait l'auditeur. Pour les prédicateurs en quête de cette juste mesure, l'optique est tout sauf un thème anodin dans les formes comparatives ; c'est un motif métatextuel qui leur permet de penser la mission même de l'éloquence sacrée et de l'amplification en particulier, comme l'a noté Stéphane Macé à propos de Bossuet :

il n'est plus question de tromper l'auditoire sur la réalité des choses, mais de restituer une grandeur et une petitesse originelles, de dévoiler, de révéler (peutêtre aussi selon l'acception chrétienne du terme). Tout est affaire de perspective ${ }^{67}$.

Il semble possible de généraliser ce constat à l'éloquence sacrée entre 1600 et 1670 . La fonction des formes de comparaison est alors théorisée par analogie avec des dispositifs de vision complexe (miroir, anamorphose), afin d'exprimer la capacité de l'éloquence sacrée à corriger la vision faussée d'une humanité rendue aveugle par le péché. Louis de Grenade compare parfois la méditation ou l'amplification à un collyre qui rend la vue à l'œil de l'âme ${ }^{68}$. Les prédicateurs emploient cette image à la coloration augustinienne pour invoquer le pouvoir de la grâce ${ }^{69}$, mais ils lui préfèrent parfois celle des instruments plus modernes qui confortent la vue. Ainsi, le télescope de la parole permet de vaincre l'éclipse du Dieu caché.

\section{Du péché comme amplification perverse du vrai}

L'amplification se voit souvent reprocher de grossir et de diminuer les choses de manière artificielle, imposant à la réalité la distorsion d'une éloquence mensongère ; en cela, elle est déplacée dans la chaire de vérité. Mais si elle rectifie un point de vue faussé, elle est légitime ; en terrain religieux, la pratique rhétorique de l'amplification semble donc présupposer le thème de l'humanité aveuglée, récurrent dans toutes les formes de l'amplification optique, qu'il s'agisse des comparaisons ou des similitudes. Nous trouvons d'ailleurs les deux alternativement sous la plume d'Étienne Molinier. En 1631, il dissèque l'œil intérieur : 
Le nerf optique de l'œil de l'ame c'est la foy, comme nous disions tantost, puis que la foy est le nerf et le soustien de la veuë spirituelle qui contemple les verités eternelles, et ce nerf se trouvant empesché par l'oubliance, et distraction ou totale, ou fort grande des choses que nous croyons, n'a plus de mouvement, et d'action pour distribuer cette veuë à l'œil du cœur, qui par ce moyen tombe dans l'aveuglement. L'humeur crystalline de cet œil spirituel c'est la pureté, et simplicité de l'intention regardant Dieu, non nous mesmes, en tout ce que nous faisons, puis que dans l'Evangile, où nostre Seigneur parle de l'œil pur, et simple, c'est à dire sans tache, qui rend tout le corps lumineux; Si oculus tuus fuerit simplex, totum corpus tuum lucidum erit ["Si votre œil est simple, tout votre corps sera lumineux »]".

Un parallèle systématique s'opère entre les causes organiques de la cécité du corps, et l'étiologie spirituelle de l'aveuglement qui touche l'âme; toutes les potentialités pédagogiques de la similitudo per conlationem se déploient alors. Mais le style du prédicateur, hors des passages explicatifs, peut se faire bien plus bref :

Mais que ferons nous contre l'esclat apparent de la vanité du monde, qui aveugle plusieurs esprits, comme la blancheur de la neige les yeux qui la regardent trop attentivement ${ }^{71}$ ?

En somme, dès 1631, l'amplification oratoire non seulement semble avoir atteint une cadence plus mesurée, mais en outre elle sait user de l'abondance et de la brièveté pour mettre en valeur une thématique aux enjeux centraux dans la culpabilisation du public : l'aveuglement spirituel accuse le péché qui en est la source ${ }^{72}$.

Qu'il soit originel ou actuel, le péché trouve son origine dans les menées du diable ou dans les mirages de l'amour-propre. Satan est en effet capable de grandir ou de diminuer l'image des choses, ainsi que l'écrit Jean-Pierre Camus en 1617 :

Le diable est un maistre tres expert en la spirituelle catoptrique, fabriquant des miroirs de specieuses apparences, qui font paroistre les objects tels qu'il veut, les petits gros, les gros petits, les esloignez proches, les proches esloignez: et c'est merveille avec ces artifices combien il abuse d'ames, en tentant il amoindrit le peché, facilite le Pardon, propose la Penitence aisée, estant perpeciee ${ }^{73}$, il l'enfle desmesurement, bouffit la Divine Justice, cache la misericorde, le tout pour faire trebuscher au desespoir ${ }^{74}$.

«Les petits gros, les gros petits » : c'est là quasiment mot pour mot la définition de l'amplification pour ses détracteurs depuis Platon $^{75}$; le démon de Camus est un diable sophiste dont l'éloquence intérieure atteint l'œil de l'âme par autant d'hypotyposes hyperboliques, que Camus représente par des miroirs grossissants ou qui amenuisent les objets. Mais c'est principalement le péché particulier qui est à la racine de tous les autres, l'amour-propre, que dénoncent les prédicateurs. Il se présente comme un miroir ou une perspective enchantée qui grossissent démesurément les mérites de l'orgueilleux à ses propres yeux :

Messieurs, les yeux de Dieu et les nostres ne peuvent s'accorder en fait de regard pour la grandeur. Ce que les uns voyent élevé, les autres le voyent abbatu: ce que ceux-ci voyent abbatu, ceux-là le considerent comme élevé. Double raison de cecy, l'une Physique, l'autre Morale. La Physique est prise d'une chose que l'experience confirme tous les jours. Regardez un objet de loin pour grand qu'il soit en soy, il paroistra fort petit à vos yeux. Pourquoy? dautant que vous le regardez de loin. Au contraire, considerez un objet de prés pour petit qu'il soit en soy, cette proximité de veuë sera capable de le grossir, et de luy donner masse à vos yeux. L'Optique en rend la raison que je n'ay pas loisir de déduire icy. Quand vous vous imaginez estre quelque chose à vos yeux, que vostre coeur s'enfle, et qu'il presume de soy; Dieu se recule de vous, et se reculant il vous regarde de loin comme un objet qui luy fait mal aux yeux, et qu'il ne peut voir de prés ${ }^{76}$. 
Ici, plus de Satan rhéteur comme chez Camus ; le démonologue cède le pas au moraliste. L'amour de soi est une autre sorte d'amplification perverse dans laquelle l'âme se persuade de sa propre excellence.

\section{De la prédication comme optique divine}

Pour lutter contre l'éloquence perverse du péché, qui présente des mirages à l'œil de l'âme, la rhétorique sacrée se construit donc comme une tentative de correction optique, qui permet de rendre aux choses leur grandeur réelle. L'image du collyre, comme celle de l'instrument de vision, est employée à propos de la rhétorique sacrée. Le point commun entre ces deux objets réside dans leur capacité à rendre la vue ; la différence, c'est que quand le collyre soigne seulement l'organe, le télescope ou le miroir déformant font voir des merveilles inouïes. L'amplification des comparaisons puise alors aux sources d'une science moderne, qui insiste sur l'opposition entre apparence et réalité. Non seulement la nouvelle optique mécaniste décrète que la lumière et la couleur sont peu ou prou des illusions, défigurant ainsi la beauté du monde ${ }^{77}$, mais elle se montre même hostile à certains thèmes religieux en dévalorisant le surnaturel. Le mathématicien et minime Jean-François Niceron, spécialiste ès illusions optiques, souligne la puissance de désenchantement propre à l'étude des miroirs, qui révèle les impostures des charlatans :

La Catoptrique ou science des miroirs nous a fait voir des productions si admirables, ou des effets si prodigieux, qu'entre ceux qui l'ont connuë et pratiquée il s'en est trouvé qui par une vaine et ridicule ostentation, ou pour abuser les plus simples, se sont efforcez de passer pour devins, sorciers ou enchanteurs comme ayant le pouvoir, par l'entremise des mauvais esprits, de faire voir tout ce qu'ils vouloient, soit passé, ou à venir. Et l'on en a veu des effets si estranges, qu'à ceux qui n'en sçavoient pas la cause, ny les raisons [...] ils devoient passer pour surnaturels, ou estre pris pour de pures illusions ou prestiges de magie diabolique ${ }^{78}$.

Quelques années plus tard, son opinion est reprise dans le cadre de l'écriture religieuse en prose par le jésuite Joseph Filère dans le Miroir sans tache:

Qui pourroit asseurer, que ces Pythonisses, ou ces renommées enchanteresses de Thessalie, ne se servoient point de cet artifice, mêlant les illusions naturelles avec les diaboliques ${ }^{79}$ ?

Prudent, l'auteur critique les sorcières thessaliennes antiques plutôt que leurs homologues du XvII ${ }^{\mathrm{e}}$ siècle ; cela suffit à mettre en évidence la valeur démystifiante de l'optique.

Ce n'est donc pas à un savoir neutre et affadi que puise l'amplification optique lorsqu'elle fait appel aux sciences de la vision, mais à un savoir démystifiant, potentiellement hostile à une perspective religieuse. Bien sûr, dans le sermon, et dans l'écriture religieuse plus généralement, l'optique ne sert plus à dénoncer les impostures des devins mais le point de vue de la chair, les manigances de la crainte ou de l'amourpropre :

On pourroit encor rendre toutes ces illusions plus affreuses, si l'on se servoit d'un miroir cylindrique concave, le tenant pendu par un filet, pour le rendre plus facilement mobile: car, comme ces miroirs rendoyent des images plus disproportionnées, et par consequent plus hideuses, elles donneroient aussi plus de terreur ; notamment, si le miroir étant mobile, ou la personne, qui est representée, prenant diverses postures, ou faisant diverses mines; celuy, qui les regarde, se trouve toûjours dans le lieu, où il faut être pour les voir; et encor qu'il n'y seroit pas toûjours si precisément, l'image paroissant et disparoissant, seroit encor plus 
capable de l'effrayer, et beaucoup plus, si elle portoit une espée nuë en main : ce, qui en a bien étonné plusieurs, qui la voyant sortir comme hors du miroir, ont été surpris de grande crainte, se croyans attaqués et blessés à l'impourveu ${ }^{80}$.

Filère emprunte ici à Giambattista della Porta un miroir effrayant qui fait surgir des figures hors du miroir, d'après un mythe scientifique très répandu depuis le Moyen Âge, et auxquels certains fondateurs de la science moderne n'ont pas manqué de s'intéresser ${ }^{81}$. Voilà pour l'illusion, voici le désenchantement :

N'est ce pas de cet artifice, que se sert ce trompeur, ennemy de nôtre salut, pour nous détourner de la vertu, par la crainte de mille vaines apprehensions, qui ne sont que des phantômes, formés dans le creux de nôtre cerveau, comme dans une chambre, où il a logé ce miroir concave, propre pour toutes ces illusions, par la foiblesse de nôtre jugement, et le peu de connoissance, que nous avons de ces ruses $^{82}$ ?

La catoptrique spirituelle, science des miroirs illusoires de l'intériorité, révèle les tréfonds de l'âme mélancolique, la manière dont ses secrets ressorts déforment le réel. Elle infléchit dans le sens de la lucidité moraliste la valeur démystifiante de l'optique moderne.

Un Dieu de vérité ne saurait être comparé aux images trompeuses des perspectives curieuses : seul l'homme est susceptible d'erreur. Ainsi, dès les années 1630, la louange $\mathrm{du}$ soleil divin laisse place insensiblement à une prédication plus moraliste, du moins d'un moraliste chrétien. En consonance avec la logique déjà notée chez Joseph Filère dans Le Miroir sans tache, l'optique sacrée des prédicateurs dénonce l'illusion. Il peut s'agir de représenter le péché comme un verre déformant pour Jean-Pierre Camus :

Le Courroux est une suffusion de sang qui faict tout paroistre rouge, yeux de Basilics, qui ne respirant que meurtres, milieu fallacieux et cholere qui transforme tout en sa passion, comme le verre coloré monstre tout l'opposite selon sa couleur ${ }^{83}$. Quelques décennies plus tard, au terme du parcours, Jean Grisel donne une assise scientifique supérieure et une plus grande cohérence au motif en évoquant les tromperies de la perspective, science des illusions :

C'est un mauvais signe, lorsque pour voir les objets on les eloigne de ses yeux. Et je hay cete sçiance trompeuze, dont l'agreable mansonge nous represante des objets sous nos yeux qui en sont extremement reculez. Je vivray, dites-vous, quarante, cinquante, soissante ans ; c'est un privilege de nôtre famille, et j'y mets fort bon ordre. Prenez garde mon ami, que vous contez peut-estre sans vôtre hôte : et vous souvenez qu'il ne tombe plus de fleurs, que les arbres ne portent de fruits. En effet c'est une verité de facile demonstration qu'il meurt plus de jeunes que de vieus; puîque dans une famile grossie de dis ou de douze Enfans, d'ordinaire il n'en reste au plus que quatre ou cinq ${ }^{84}$.

Le Créateur lumineux laisse place à l'image d'un "Dieu chirurgien » (Benedetta Papasogli ${ }^{85}$ ) qui sonde les âmes et les cœurs et les guérit par la grâce, telle que la met en scène l'optique moraliste d'un Nicole par exemple.

\section{Il cannochiale tridentino. Le télescope de la prédication}

Ainsi, l'amplification, en révélant les mirages du péché, rend à l'âme ses capacités de droite vision et de connaissance dans la réalité. Ce discours moraliste doit finalement déboucher, sous peine d'impasse, sur la vision tant attendue ; après avoir pansé l'œil blessé de l'âme, les prédicateurs évoquent les spectacles qu'il observe, de même que l'objet qu'il permet d'atteindre :

Cette veuë intellectuelle de l'ame raisonnable estant assistee de la revelation divine, corroboree de la grace du sainct Esprit, eslevée par la lumiere de la foy void, et 
regarde de loin les choses eternelles, ou l'homme doit aspirer; et comme certaines lunetes approchent de l'œil corporel les especes des objects esloignez, où il ne pourroit atteindre sans ce secours, la mesme lumiere de la foy approche de l'œil de l'entendement ce qui sans ceste veuë seroit éloigné par une distance infinie de sa naturelle portée; car il atteint par son moyen de la terre au ciel, du temps à l'Eternité, du present au futur, du visible à l'invisible, et voit ce qui surmonte l'esprit et la pensée ${ }^{86}$.

Représentant d'une prédication à tendance conceptiste ${ }^{87}$, Étienne Molinier compare de manière très éclairante le télescope qui grossit les objets à la foi, don de Dieu qui permet de voir les réalités cachées aux sens. En ces lignes, il est bien question d'amplification ; Molinier reprend l'idée du grossissement associé à ce procédé, en lui donnant une coloration scientifique: le télescope fait des objets célestes les plus lointains nos voisins. On pourrait objecter que le prédicateur parle ici de la théologie et de la conversion en général, plutôt que de l'amplification oratoire. Pourtant, si l'on considère d'une part que l'orateur sacré est utilisé par Dieu comme instrument pour appeler à la conversion (selon la théorie du Christus orator, fondatrice des rhétoriques borroméennes ${ }^{88}$ ), et si, d'autre part, l'amplification reste d'après Louis de Grenade l'une des ressources les plus essentielles dont dispose le prédicateur tridentin, il faut en conclure que la métaphore du télescope évoque aussi tacitement l'effet de la prédication sur l'esprit de l'auditeur ; ce résultat n'est jamais dans le christianisme de l'ordre de la pure conviction, mais dépend toujours de la grâce divine. Ainsi, de même que le Christ guérit l'aveugle-né avec un peu de boue, Molinier entend dessiller ses contemporains, en leur rendant accessibles par la parole les perfections divines qui échappent à leur pensée. La prédication, si nous lisons le texte dans cette optique, serait donc un moyen d'action privilégié de la grâce, en cela même qu'elle use d'une rhétorique déformante. Il n'est donc pas étonnant que le télescope soit compris comme un symbole de la juste rhétorique, comme il l'est encore pour Tesauro en 1654.

Si l'on s'intéresse à présent à la mise en œuvre de ce télescope de la parole pointé vers Dieu ou l'Univers, la notion de point de vue y tient une place prépondérante. Le traitement textuel du motif peut l'unifier ou le démultiplier, signant là deux formes d'amplification différentes. Une première version considère que l'Infini divin se découvre en de multiples reflets. Nicolas Caussin amplifie l'idée de la vision de Dieu à travers le miroir de la nature par cette belle similitude :

L'empereur Domitien se fist autrefois une sale toute lambrissée de miroirs où de tous costez on voyoit son visage, mais le temps en cassa bien-tost l'original, les figures et le plan. Il n'y a que cet Univers qui nous paroist comme la salle du premier Monarque du monde, qui nous porte dans les yeux ses pourtrais, qui renaissent continuellement en tant d'ouvrages, et se fait voir par tous les siecles ${ }^{89}$.

Les phengites sont ces pierres dotées de capacités réfléchissantes que le méfiant Domitien avait placées dans son palais pour espionner son entourage, d'après Suétone ${ }^{90}$. Sous la plume du Jésuite, elles multiplient l'image du Créateur comme celle de jadis représentaient l'empereur. Comme ces miroirs sont disposés selon des angles de réflexion divers, ils rendent différentes images qui révèlent chacune une facette de Dieu. Ce n'est plus le soleil divin qui est perçu, mais les multiples facettes d'une divinité infinie, qui ne saurait être entièrement révélée par aucun point de vue. Le lecteur songe alors à " l'Omnivoyant » du Tableau de Nicolas de Cues, ce Dieu infini qui porte partout son regard et qui est vu de tous ${ }^{91}$. Mais si Caussin parle de contemplation de la nature, dans les décennies suivantes, le motif des miroirs multiples subit une évolution dans un sens bien plus favorable à la foi qu'à la raison : 
S. Paul dit, que la foy est un miroir. Mais il faut-adjoûter que c'est un miroir à plusieurs faces, dans lesquelles nous trouvons toujours Jesus Christ, représenté sous de differentes figures ${ }^{92}$.

Jacques Biroat brode ici peut-être sur la longue tradition des miroirs théâtraux, ancêtres des labyrinthes de miroirs des fêtes foraines : il s'agit de glaces qui, mises face à face dans un meuble ou une même pièce, multiplient les objets à l'infini en les montrant sous divers angles ${ }^{93}$. L'amplification ne se fait plus tant par la copia que par un motif frappant, très fort et très signifiant. Mais là où Caussin vantait la nature, c'est vingt ans plus tard la foi qui est le principe d'une « Optique chrétienne ${ }^{94}$ ».

Un deuxième dispositif suppose que les points de vue sont multiples mais qu'un seul révèle l'essence ; c'est le cas dans l'anamorphose à miroir du premier «Sermon de la Providence » de Bossuet, prononcé à Dijon en 1656 :

la première vue ne vous montre qu'une peinture qui n'a que des traits informes et un mélange confus de couleurs : mais sitôt que celui qui sait le secret vous le fait considérer par le point de vue ou dans un miroir tourné en cylindre qu'il applique sur cette peinture confuse, aussitôt, les lignes se ramassant, cette confusion se démêle, et vous produit une image bien proportionnée. Il en est ainsi de ce monde : quand je le contemple dans sa propre vue, je n'y aperçois que désordre ; si la foi me le fait regarder par rapport au jugement dernier et universel, en même temps j'y vois reluire un ordre admirable ${ }^{95}$.

A priori, à quelques années d'écart, il semble y avoir une contradiction manifeste entre l'approche de Bossuet et celle de Biroat. Celui-ci valorise la multiplicité des points de vue dans sa pratique de l'amplification, tandis que Bossuet reprend un dispositif où, si deux visions $\mathrm{du}$ dessin sont possibles, une seule importe, celle qui s'opère dans le miroir. Le mystère s'éclaircit si l'on remarque quel objet est considéré dans chaque texte. Biroat parle de contempler le divin qu'aucune vision adéquate ne peut saisir, d'où la pluralité des points de vue. Bossuet parle quant à lui de la Providence, c'est-à-dire du monde lui-même envisagé sub specie ceternitatis. Et la vérité du cosmos ne peut qu'être une au regard de Dieu, en tant que la nature n'est que le produit de son action créatrice. En somme, comme au temps des doctes similitudes rapprochant l'astre du jour du soleil de justice, l'amplification opère une transition de la pluralité des choses existantes à l'Unité divine; mais les prédicateurs sont plus attentifs au caractère inexprimable et insaisissable de celle-ci, et accordent plus d'importance à la manière dont l'inconnaissance humaine peut l'approcher.

\section{Conclusion. Des orbes de Christophe Scheiner à l'ermite de Manresa}

31 Les deux modes principaux de l'amplification par comparaison soulignent donc, sur fond de dissemblance, les quelques analogies qui relient le visible et l'invisible; ce procédé rhétorique se légitime comme soutien à une humanité dont l'œil est blessé par le péché originel. Pour extraire la quintessence de notre propos, nous emprunterons une image au minime d'origine bourguignonne Adrien Roussel, lecteur en théologie. Celui-ci fait paraître à Munich en 1646, en latin, une Optica christiana... (voir note 94) : une Optique chrétienne, ou Eil du verbe incarné. Sous ce titre étrange, il propose une vaste méditation sur la vie du Christ, qui insiste notamment sur ses moments les plus humbles, le lavement des pieds ou encore la mort sur la Croix. Roussel met en œuvre tout un ensemble de jeux optiques pour amplifier les articulations majeures de son propos. Ainsi, la chambre noire signale l'étrangeté de la vie du Christ qui passe par la 
mort pour aller à la gloire, tout en montrant que les deux étapes sont indissociablement liées :

Cette belle lumière qui vient dans le Monde le fait dans une position inversée, ce qu'il est plaisant d'expliquer par un autre emblème du même Scheiner, dans lequel la lumière vêtue des espèces des couleurs s'engageant en entier dans un lieu très obscur, à travers une fissure, est transportée à travers un disque [orbis] cristallin très pur, à la manière d'une pyramide lumineuse, après que les images des choses ont été conduites de bas en haut par le véhicule de la lumière, en position inversée. Ensuite, ces images sont redressées, après qu'un second cristal a été ajouté à la distance requise, avec cette inscription : Post eversionem erigor [« Après l'inversion je suis redressé »]. ô admirable inversion de la divine Sagesse, tant qu'elle apparaitt dans le pertuis étroit de la terre ! [...] Ainsi les Philosophes de l'Antiquité estimaient que toute la sagesse christique était une science contradictoire. Qui serait d'avis que le chemin des richesses passe par la pauvreté, celui de la gloire par l'humilité, celui de la souveraineté par la croix, celui de l'immortalité par la mort ${ }^{96}$ ?

Dans la camera oscura ainsi décrite, le premier orbe inverse l'image, le second la redresse. Le lecteur se souvient peut-être que la chambre noire est, dans la nouvelle optique depuis Johannes Kepler (Paralipomènes à Vitellion, 1604) et le jésuite Christophe Scheiner (Oculus, 161997) cité par Roussel, le modèle de l'œil, dans lequel les images s'inversent avant d'atteindre la rétine, pour être ensuite redressées par le cerveau ${ }^{98}$, comme le prouve Descartes en 1637. C'est dire à quel point la chambre noire symbolise la science de la vision, telle que la comprennent les Modernes. Sur un plan rhétorique, le jeu de ces deux orbes mystérieux semble symboliser à la perfection la logique de l'amplification dans l'optique post-tridentine. Si la vérité divine est renversée en folie par le monde ignorant, l'optique du Verbe incarné - notamment dans la rhétorique sacrée - lui rend son lustre initial. Comme le souligne Stéphane Macé, la pratique religieuse de l'amplification met alors en évidence la nature fondamentalement contradictoire de l'homme, tiraillé entre sa misère présente et sa destinée future ${ }^{99}$.

La chambre noire résume ainsi la logique de la rhétorique chrétienne, car la déformation d'une déformation restaure la vérité dans son état initial : la vérité divine doit passer par les vertus de l'amplification, capable de grossir ou d'amenuiser les choses, pour être justement perçue par une humanité pécheresse, de même que le Christ doit passer par la mort pour ressusciter - autre passage que Roussel illustre, dans le même chapitre, par la chambre noire. L'artifice rhétorique est légitimé par la corruption du péché originel, source d'aveuglement spirituel. Il n'en résulte aucune vision tragique, mais plutôt l'impression que le Dieu caché peut se contempler sereinement dans le miroir de l'âme, une fois qu'ont été levés les obstacles de l'orgueil. Au Gesù, un des vitraux d'Andrea Pozzo (1642-1709), célèbre perspecteur et artiste jésuite, représente Ignace de Loyola en ermite pendant sa retraite à Manresa. Le saint, habillé pauvrement, prie dans une grotte, entouré des accessoires habituels des solitaires chrétiens, des crânes aux fouets, en passant par les livres. Or, c'est dans ce dénuement et cette solitude qu'il est favorisé d'une vision céleste de la Trinité. Sur le haut du vitrail, un emblème qui représente une chambre noire porte une devise paradoxale : In obscuro miranda relucent ${ }^{100}$, des merveilles reluisent dans l'obscurité. La devise pourrait aussi être celle des prédicateurs tridentins intéressés par l'optique, et en particulier des plus sénéquiens d'entre eux. 


\section{NOTES}

1. Quintilien, Institution oratoire, texte établi et traduit par J. Cousin, Paris, Les Belles Lettres, coll. «CUF », 2003, VIII, 4, 9-10. S. Macé, «L'amplification dans le Carême du Louvre » dans Styles, genres, auteurs, $\mathrm{n}^{\circ} 2$, textes réunis par Anne-Marie Garagnon, Paris, Presses Universitaires de Paris-Sorbonne, 2002, p. 37-38.

2. Pierre de Besse (1567-1639), prédicateur de Condé et du roi, originaire du Limousin, se fit une réputation de gloire de l'éloquence sous Henri IV et Louis XIII. Gaspard de Séguiran (1569-1644), jésuite, eut du succès par ses prédications à la Cour dans les années 1610 et 1620 .

3. André de l'Auge, de l'Ordre des Frères mineurs, professeur de théologie à Nancy vers 1620 ; Étienne Molinier (1580-1647), docteur en droit et prédicateur toulousain; Jean Le Jeune (1595-1672), missionnaire oratorien actif dans le sud du Royaume; Léon de Saint-Jean (1600-1671), dignitaire carme, proche de Richelieu et de Mazarin, auteur d'une encyclopédie d'esprit renaissant.

4. Peter Bayley, French Pulpit Oratory (1598-1650), Cambridge, Presses Universitaires de Cambridge, 1980, p. 149.

5. Sur le sujet, on peut consulter les ouvrages suivants: C. Mouchel, Cicéron et Sénèque dans la rhétorique de la Renaissance, Margburg, Hitzeroth, coll. «Ars rhetorica » (3), 1990 ; M. Blanco, Les Rhétoriques de la Pointe. Baltasar Gracián et le Conceptisme en Europe, Paris, Champion, 1992 ; P. Laurens, «Entre la poursuite du débat sur le style et le couronnement de la théorie de l'“actio" : Vossius et le réaménagement de l'édifice rhétorique », dans M. Fumaroli dir., Histoire de la Rhétorique dans l'Europe moderne (1450-1950), Paris, PUF, 1999, p. 499-500.

6. Jacques-Bénigne Bossuet, Le Carême du Louvre, éd.C.Cagnat-Debœuf, Paris, Gallimard, coll. «Folio classique », 2001, p. 114-115. S. Macé, "L'anamorphose dans le Carême du Louvre de Bossuet (1662) », dans L'optique des moralistes, Actes du colloque international de Grenoble (mars 2003), dir. Bernard Roukhomovsky, Paris, Champion, 2005, p. 405-418.

7. R. Zuber, Les « belles infidèles» et la formation du goût classique (1968), Paris, Albin Michel, 1995, p. 64.

8. Gaspard de Séguiran, Sermons sur la parabole de l'enfant prodigue, Paris, Nicolas du Fossé, 1612, p. 307.

9. Quintilien, op. cit., VIII, 4, 9: «Pourtant si cette amplification tend à aller plus haut, celle qui procède par comparaison tire le grossissement d'éléments moins importants. En exagérant en effet ce qui est inférieur, on élève nécessairement ce qui est au-dessus. " Jean de Richesource, L'Éloquence de la chaire ou la Rhétorique des prédicateurs, Paris, À l'Académie des Orateurs, 1675, p. 165-166.

10. Pierre de Besse, Premieres conceptions theologiques sur le Caresme, Paris, Nicolas du Fossé, 1604, fol. 328a.

11. Nicolas Caussin, Eloquentice sacræe et humance parallela, libri XVI, Paris, S. Chappelet, 1619, liv. IV, chap. LIII, p. 173.

12. Érasme, De duplici copia verborum ac rerum, Lyon, apud Johannem Tornæsium, 1558, p. 213. François de Sales, Traité de la prédication (1604), dans Euvres complètes de Saint François de Sales, Paris-Béthune, s. n., 1836, chap. III, p. 10. Voir aussi sur le même sujet Jean de Richesource, qui dans L'Éloquence de la chaire ou la Rhétorique des prédicateurs (op. cit., p. 167) parle de "presser l'allusion » dans le parallèle d'une similitude.

13. G. de Séguiran, "Sermon pour le second dimanche de Caresme ", Sermons doctes et admirables sur tous les jours de Caresme, et Feriees de Pasques. Preschez à Paris par un celebre personnage de nostre temps, Paris, Nicolas du Fossé et Pierre Chevallier, 1613, p. 233-253.

14. Pierre de Besse, « Pour le Samedy d'apres le $4^{\mathrm{e}}$ Dimanche de Caresme », op. cit., fol. 325b-340a. 
15. Rhétorique à Herennius, trad. G. Achard, Paris, Les Belles Lettres, coll. «CUF », 1997, IV, 49, p. 208. Louis de Grenade, La Réthorique [sic] de l'Eglise ou l'Eloquence des predicateurs (1576), traduction de Nicolas-Joseph Binet, Paris, J. Villette, 1698, liv. v, p. 231-234 (ce texte est édité dans ce numéro, section « Atelier »).

16. Depuis l'Antiquité, et à nouveau chez Grenade, la similitudo per conlationem est également connue pour «mettre la chose devant les yeux » (ante oculos ponere) selon la formule consacrée (op. cit., p. 632-633).

17. Pierre de Besse, Conceptions theologiques sur les quatre fins de l'homme. Preschées en un Advent l'an 1605, Paris, Nicolas du Fossé, 1606, vol. I, p. 632. La citation latine vient de saint Paul (Épître aux Galates, V, 17). Nous reprenons la traduction de Lemaitre de Sacy, qui date de 1667 pour le Nouveau Testament (La Bible, éd. Ph. Sellier, Paris, Robert Laffont, 1990).

18. Le chanoine berrichon J. Barion (L'Iris sacré, Paris, Jean de Freval, 1619), inspiré par les prédications de G. de Séguiran, développe ainsi en un style très descriptif les beautés de l'arc-enciel, assimilé aux trois vertus théologales.

19. Pierre de Besse, Conceptions theologiques sur les quatre fins de l'homme, loc. cit. Pour Saint Augustin (La Cité de Dieu, xx, 14), nous reprenons les CEuvres complètes de Saint Augustin, traduites pour la première fois en français sous la direction de M. Poujoulat et de M. l'abbé Raulx, Bar-le-Duc, Louis Guérin, 1864-1873, t. IV, p. 462 : « une vertu divine [...] en un instant, afin que sa conscience le condamne ou le justifie, et qu'ainsi tous les hommes soient payés en un moment. »

20. Lucrèce, De la nature/De rerum natura, traduction et présentation par J. Kany-Turpin, Paris, Garnier-Flammarion, 1993, liv. II, v. 110-124, p. 120.

21. Voir F. Libral, Le Soleil caché, rhétorique sacrée et optique au XVII siècle en France, Paris, Classiques Garnier, à paraître dernier tr. 2014, chap. II.

22. Rhétorique à Herennius, op. cit., IV, 60, p. 209. Louis de Grenade, op. cit., liv. v, p. 232.

23. Gaspard de Séguiran, Sermons sur la parabole de l'Enfant prodigue, op. cit., p. 308.

24. Il semble possible d'opérer un rapprochement entre la similitudo per brevitatem des traités latins et ce que Peter Bayley appelait la catenary prose. Voir P. Bayley, op. cit., p. 85.

25. Aristote, Rhétorique, trad. M. Dufour et A. Wartelle, Paris, Les Belles Lettres, coll. "CUF », 1973, III, 2, 1405a11, p. 43. Voir aussi la question de la «métaphore par similitude» ou par analogie chez Emmanuele Tesauro et Baltasar Gracián (dans M. Blanco, op. cit., p. 261 et p. 384).

26. Étienne Molinier, Sermons pour tous les dimanches de l'année (1631), Toulouse, Arnaud Colomiez, 1639, p. 411.

27. C. Mouchel, op. cit., p. 160.

28. André de l'Auge, La Saincte Apocatastase. Sermons adventuels sur le Psalme XXIIX, Paris, Robert Foüet, 1623, p. 826.

29. S. Macé, « L'obscurité et les théories rhétoriques de l'amplification », dans L'Obscurité, Langage et herméneutique sous l'Ancien Régime, dir. D. Denis, Louvain-La Neuve, Academia Bruylant, coll. « Au cœur des textes » ( $n^{\circ}$ 9), 2007, p. 55-56.

30. Jean Le Jeune, "Sermon des trois naissances du fils de Dieu pour le jour de Noël ", Le Missionnaire de l'Oratoire. Sermons de la foy, $6^{e}$ partie, Rouen, Richard Lallemant, 1671, p. 872. Dans la suite du sermon, Le Jeune utilise des similitudes pour prouver son propos, illustrant ainsi la complémentarité des deux formes.

31. Première épître à Timothée, VI, 16.

32. L'amplification sénéquienne apporte ainsi un "point de vue original ", comme le précise C. Mouchel à propos de l'amplification per ratiocinationem (op.cit., p.113) et de sa capacité à exprimer les différents aspects d'une idée chez Grenade (ibid., p. 115).

33. Rodolphe Agricola, De inventione, Strasbourg, apud Johannem Knoblouchum, 1521, fol.36b. Voir T. Cave, Cornucopia. Figures de l'abondance au XVI' siècle: Érasme, Rabelais, Ronsard, Montaigne (1979), Paris, Macula, coll. « Argô », 1997, p. 42. 
34. Peter Bayley, op. cit., p. 149.

35. M.Blanco, op. cit., p. 83sq. Delphine Denis («Approches de l'obscurité au siècle classique», dans L'Obscurité, Langage et herméneutique sous l'Ancien Régime, op. cit., p. 55-67) souligne toutefois les valeurs positives que peut prendre la notion à l'époque classique, jouant des potentialités brillantes offertes par l'équivoque et les suppositions.

36. Quintilien, op.cit., V, XI, 5 sq., et part. 6, p. 164 (« Il faut donc examiner si la similitude est totale ou partielle, afin de l'appliquer dans sa totalité ou seulement pour la part qui sera utile.»). 37. Léon de Saint-Jean, Le Portrait de la Sagesse universelle, Paris, G. Bénard et A. Pasdeloup, 1655, t. II, « Le Sanctuaire de l'éloquence », p. 425.

38. S. Macé, «L'amplification dans le Carême du Louvre », op. cit., p. 37.

39. Léon de Saint-Jean, La France convertie: octave a l'honneur du B. S. Denys l'Areopagite, premier evéque de Paris, protecteur du royaume, patron des roys, Paris, Florentin Lambert, 1661, p. 82-83.

40. Léon de Saint-Jean, Les Divins paradoxes de l'eucharistie, octave du Tres-saint Sacremant de l'autel, préchée en l'Eglise principale de Bruxelles, Bruxelles, Jean Mommart, 1663, p. 8.

41. Sur l'antithèse et la métaphore combinées en un mode unique d'amplification, nous renvoyons aux analyses éclairantes de Peter Bayley sur le panégyrique de Sainte Lucie de Molinier (op. cit., p. 126-127).

42. Léon de Saint-Jean, La France convertie, op. cit., p. 95.

43. Claude Hopil, Les Divins Eslancemens d'amour exprimés en cent cantiques spirituels faits en l'honneur de la Tres-saincte Trinité, Paris, Sebastien Huré, 1629, cantique XI, str. II, p. 43 : «Je voy le Dieu de Dieu, dans une claire nuë / Le Soleil est caché ».

44. C. Mouchel, op. cit., p. 330.

45. Léon de Saint-Jean, Les Divins paradoxes, op. cit., p. 15.

46. Florent Libral, op. cit., chap. VI.

47. Docteur en théologie et prédicateur du roi.

48. Jacques Biroat, La Condamnation du Monde par le Mystere de l'Incarnation du fils de Dieu, Paris, Edme Couterot, 1662, p. 30-31.

49. Léon de Saint-Jean, La France convertie, op. cit., p. 95-96. La citation latine vient de l'Ancien Testament (Amos, VIII, 9). Nous reprenons la traduction de Lemaître de Sacy (La Bible, op. cit.), qui date de 1679 pour les livres des Douze Petits Prophètes.

50. Emmanuele Tesauro, Il cannocchiale aristotelico, présentation bilingue d'extraits choisis avec traduction sous le titre La métaphore baroque par Y. Hersent, Paris, Seuil, 1999, p. 67 sqq.

51. Voir C. Mouchel, op. cit., p. 211 : «Il en va des concepts comme des choses divines. Lorsqu'on croit les saisir par une image ressemblante, on ne possède jamais qu'une image dissemblable dans la ressemblance, mais l'illusion de la similitude nous porte à croire que ce concept adhère parfaitement à son signe et que par là nous accédons à l'être: c'est l'erreur constante du cicéronianisme. Le passage par le voile, celui des images expressément dissemblables pour les choses divines, celui d'une révélation inachevée pour les concepts, est le moyen d'accès qu'impose la faiblesse humaine, mais où se révèle en même temps le pouvoir de la subtilitas, vis intellectus, qua difficilia cognitu facile comprehenduntur [la subtilité, cette puissance intellectuelle par laquelle les choses difficiles sont facilement comprises]. »

52. Thomas Giroult, L'Aigle françois, ou Conceptions divines sur le Premier Chapitre de Sainct Jehan preschées en un Advent, Paris, Sébastien Cramoisy, 1611, p.80-81. La citation de Basile (et sa traduction) provient de Basile de Césarée, Contre Eunome, introduction, traduction et notes de B. Sesboüé, Paris, Cerf, coll. « Sources chrétiennes » (305), 1983, t. II, p. 65.

53. Pseudo-Denys, La Hiérarchie céleste. CEuvres complètes, éd. M. de Gandillac, Paris, Aubier, 1943, p. 189.

54. J. Filère, Le Miroir sans tache enrichy des Merveilles de la nature dans les miroirs, rapportées aux effets de la grace, Lyon, veuve de Claude Rigaud, et Philippe Borde, 1636, p. 517-518.

55. Ibid., p. 518. 
56. Charles Hersent, Le Scandale de Jesus Christ dans le Monde. Presché par le sieur Hersent dans l'Eglise de sainct Gervais le second dimanche des Advens, en la presence de Monseigneur le Coadjuteur de Paris, s. 1., s. n., 1644, p. 10-11.

57. Pierre Blanchot, Le Vray Accomplissement des desirs de l'homme, Paris, Sebastien Cramoisy, 1635, p. 125-126.

58. Première épittre aux Corinthiens, I, 18-31.

59. C. Mouchel, op. cit., p. 95.

60. Pour ces points qu'il serait trop long de développer ici, voir F. Libral, op. cit., chap. II et IV.

61. Pierre de Besse, Premieres conceptions theologiques sur le Caresme, op. cit., fol. 327a.

62. Jean Grisel, Sermons sur tous les Evangiles du Caresme. Par le R. P. Jean Grisel, de la Compagnie de Jesus. Tome premier, Paris, Denis Bechet et Louis Billaine, 1658, p. 782.

63. J.-R. Armogathe, "Lueurs et ténèbres du Dieu caché », dans Le Dieu caché: les peintres du Grand Siècle et la vision de Dieu. Exposition de l'Académie de France à Rome 19 octobre $2000-28$ janvier 2001, textes réunis par O. Bonfait et N. Mac Gregor, Rome, Edizioni de Luca, 2000, p. 17-25.

64. Charles Roussel, L'Ouverture des sept sceaux faite par les sept cornes de l'Agneau immaculé Jesus, pour les sept Festes principales de la Vierge sa tres-digne Mere, Paris, Denis Moreau, 1627, p. 429.

65. Léon de Saint-Jean, La France convertie, op. cit., p. 83.

66. S. Macé, «L'obscurité et les théories rhétoriques de l'amplification », op. cit., p. 66.

67. S. Macé, « L'amplification dans le Carême du Louvre », op. cit., p. 28.

68. Louis de Grenade, Année ecclesiastique, ou Sermons du Reverend Pere Louis de Grenade, t. II, Paris, Claude Herissant, 1697, p. 214.

69. Saint Augustin, "Traité II " sur l'Évangile de Jean, dans CEuvres complètes de Saint Augustin, op. cit., Bar-Le-Duc, Louis Guérin, 1864, t. x, p. 329. Cette citation est reprise dans la sylve de lieux communs de Grenade (Euvres complètes, traduction de l'abbé Bareille, Paris, Louis Vivès, 1865, vol. Xx, p. 72).

70. É. Molinier, Sermons pour tous les dimanches de l'année, op. cit., p. 437-438. La citation latine vient de l'Évangile selon Saint Mathieu (VI, 22). Nous reprenons la traduction de Lemaître de Sacy (La Bible, op. cit.), qui date de 1667 pour le Nouveau Testament.

71. É. Molinier, op. cit., p. 442.

72. J. Delumeau, Le Péché et la Peur. La culpabilisation en Occident, XIII'-XVIII ${ }^{e}$ siècles, Paris, Fayard, 1983, p. 275.

73. Perpeciee : latinisme, de perpetior (« souffrir jusqu'au bout »).

74. Jean-Pierre Camus, Homelies sur la passion de Nostre Seigneur (1617), Paris, Claude Chappelet, 1623, p. 391-392.

75. Platon, Phèdre, 267a, traduction d'É. Chambry, Paris, Garnier-Flammarion, 1964, p. 153 : « [...] Tisias et Gorgias, eux qui ont découvert que le vraisemblable est bien supérieur au vrai, qui, par la force de leur parole, font paraître grand ce qui est petit et petit ce qui est grand [...].»

76. Jean Grisel, Baltazar ou l'oubly de Dieu puny en la personne de ce dernier Roy de Babylone. Advent Presché à Paris, en la Paroisse de S. Eustache, l'année 1640, Paris, Denis Bechet, 1655, p. 265-266.

77. Pierre Ango, L'Optique divisée en trois livres, Paris, Estienne Michalet, 1682, p. 4-5.

78. Jean-François Niceron, La Perspective curieuse du R.-P. Niceron, Minime [...] avec l'Optique et la Catoptrique du R. P. Mersenne [...] du même ordre, mise en lumière après la mort de l'auteur, Paris, J. du Puis, 1663, liv. III, avant-propos, p. 147.

79. J. Filère, op. cit., p. 59.

80. Ibid., p. 60.

81. J. Baltrušaitis, Le Miroir, révélations, science-fiction et fallacies, Paris, Elmayan-Le Seuil, 1978, p. 228.

82. J. Filère, op. cit., p. 60. 
83. Jean-Pierre Camus, Premieres homelies quadragesimales de Messire Jean Pierre Camus, Evesque et Seigneur de Bellay, Paris, Claude Chappelet, 1615, p. 88-89.

84. Léon de Saint-Jean, Les Triomphes Evangeliques. Caresme presché dans l'Eglise Saint Germain de l'Auxerrois, Paris, Jean Du Puis, 1665, t. I, p. 298.

85. B. Papasogli, «Le modèle anatomique dans l'œuvre de Pierre Nicole », Littérature et anatomie, Actes du colloque de l'Association Internationale des Études Françaises, CAIEF, nº 55 (2003), p. 343. 86. É. Molinier, Sermons pour tous les dimanches de l'année, op. cit., p. 423.

87. P. Bayley, op. cit., p. 94-95.

88. Marc Fumaroli, L'Âge de l'éloquence. Rhétorique et "res litteraria" de la Renaissance au seuil de l'époque classique (1980), Genève, Droz, 2002, p. 140.

89. Nicolas Caussin, Le Buisson ardent, figure de l'Incarnation, contenant vingt-quatre discours sur les Mysteres de l'Advent, Paris, Jean du Bray, 1647, p. 174.

90. Suétone, Vie des douze Césars, Domitien, texte traduit et établi par H. Ailloud, Paris, Les Belles Lettres, coll. "CUF », 1964, XIV, p. 94: «il fit disposer sur les murs des portiques où il avait coutume de se promener des plaques de phengite, dont la surface brillante devait lui permettre de voir par réflexion tout ce qui se passait derrière lui. » Il s'agissait d'après H. Ailloud d'une sorte d'une pierre brillante de Cappadoce, semblable au marbre et mentionnée aussi par Pline.

91. Nicolas de Cues, Le Tableau ou la vision de Dieu, trad. A. Minazolli, Paris, Cerf, 1986.

92. J. Biroat, La Condamnation du Monde par le Mystere de l'Incarnation du fils de Dieu. Preschee durant l'Advent dans l'Eglise de S. Germain l'Auxerrois, l'Année 1660, Paris, Edme Couterot, 1662, p. 18.

93. F. Libral, «Optique, perspective et rhétorique religieuse : l'indivision des savoirs au cœur de la reconfiguration d'une alliance séculaire ", "L'indivision des savoirs » en question, Littératures, $\mathrm{n}^{\circ}$ 67 (2012), numéro dirigé par F. Népote, p. 85-86.

94. D'après le titre d'Adrien Roussel (Optica christiana, sive Verbi incarnati Oculus in obscurioribus fidei divinae mysteriis, Munich, D. Straubium, 1646).

95. Jacques-Bénigne Bossuet, Cuvres oratoires de Bossuet, édition critique complète par J. Lebarq, Lille-Paris, Desclée de Brouwer, 1890-1897, t. II, p. 155.

96. Adrien Roussel, op. cit., p. 10-11. Nous traduisons.

97. Roussel renvoie au frontispice de l'Oculus, hoc est fondamentum opticum (Innsbruck, apud Danielem Agricolam, 1619), qui reproduit plusieurs emblèmes, dont celui qu'il décrit.

98. G. Simon, Archéologie de la vision, Paris, Seuil, 2003, p. 223 sq., « En réponse à Kepler, la théorie cartésienne de la vision ».

99. S. Macé, «L'amplification dans le Carême du Louvre », op. cit., p. 38.

100. G. Sale dir., L'Art des Jésuites, Paris, Mengès, 2003, p. 18. S. Silvestri, «In obscuro miranda relucent. Les vitraux du couloir du collège des Jésuites à Rome ", Vitrocentre Romont : Les panneaux de vitrail isolés. Die Einzelscheibe. The single stained-glass panel. Actes du XXIve Colloque International du Corpus Vitrearum (Zurich 2008), Bern, Berlin, Bruxelles, Francfort, New York, Oxford, Vienne, Peter Lang, 2010, p. 181-190.

\section{AUTEUR}

\section{FLORENT LIBRAL}

Université de Toulouse-II - ELIRE 